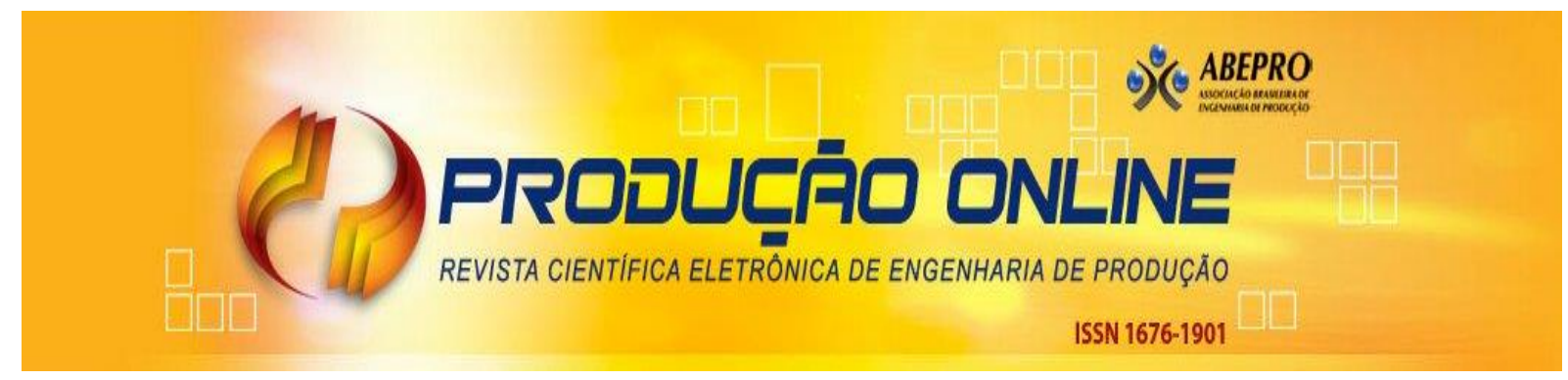

\title{
PROGNÓSTICO DA GESTÃO DOS RESÍDUOS SÓLIDOS URBANOS DE UMA CIDADE DE MÉDIO PORTE: ESTIMATIVA DE IMPACTOS AMBIENTAIS
}

\section{PROGNOSIS OF THE MUNICIPAL SOLID WASTE MANAGEMENT OF A MEDIUM CITY: ENVIRONMENTAL IMPACTS ESTIMATION}

\author{
Luiz Neto Paiva e Silva Muller* E-mail: luizpsmuller@gmail.com \\ João Bosco Furtado Arruda** E-mail: barruda@glen.ufc.br \\ Rosane Lúcia Chicarelli Alcantara*E-mail: rosane@ufscar.br \\ Lucas Rodrigues Deliberador* deliberadorlucas@gmail.com \\ *Universidade Federal de São Carlos (UFSCAR), São Carlos, SP \\ **Universidade Federal do Ceará (UFC), Fortaleza, CE
}

Resumo: A busca por um sistema de gestão de Resíduos Sólidos Urbanos (RSU) sustentável é um desafio para gestores municipais. Para isso, é necessário inicialmente entender o atual sistema de gestão e seus futuros resultados. Este trabalho teve o objetivo de realizar o prognóstico do atual sistema de gestão de RSU do município de Juazeiro do Norte no Ceará. O estudo é quantitativo e utilizou métodos quantitativos de modelagem para estimar, em um período de 20 anos (2016 a 2035), impactos ambientais de ocupação de área por aterro e de emissão de gás metano $\left(\mathrm{CH}_{4}\right)$ e de gás carbônico $\left(\mathrm{CO}_{2}\right)$. Os resultados apontam que, caso nada seja feito, os RSU coletados e enterrados no aterro do município, entre 2016 e 2035, ocuparão uma área equivalente a 20 campos de futebol $\left(147.140 \mathrm{~m}^{2}\right)$ e emitirão aproximadamente 153.300 e 420.600 toneladas de $\mathrm{CH}_{4}$ e $\mathrm{CO}_{2}$, respectivamente, que se estenderão por 40 anos. Os resultados dessa pesquisa destacam um cenário futuro preocupante que pode e deve ser mitigado através do planejamento e implementação de alternativas de mudanças do atual sistema de gestão de RSU da região.

Palavras-chave: Resíduos sólidos urbanos. Prognóstico. Impactos ambientais. Efeito estufa. Juazeiro do Norte.

\begin{abstract}
The search for a sustainable Municipal Solid Waste (MSW) management is a challenge for managers. Initially, it is necessary to understand the current management system and its future results. This work aims to realize the prognosis of the current MSW management system of the municipality of Juazeiro do Norte in Ceará. This study used quantitative modeling methods to estimate the environmental impacts of land use by landfill and the emission of methane $\left(\mathrm{CH}_{4}\right)$ and carbon dioxide gas $\left(\mathrm{CO}_{2}\right)$ over a period of 20 years (2016 to 2035). The results indicate that the MSW collected and buried in the landfill between 2016 and 2035, will occupy an area equivalent to 20 soccer fields $(147,140$ $\mathrm{m}^{2}$ ) and will emit approximately 153,300 and 420,600 tons of $\mathrm{CH}_{4}$ and $\mathrm{CO}_{2}$, respectively, which will last 40 years. This future scenario should be mitigated through the planning of alternatives for changes in the region's current MSW system.
\end{abstract}

Keywords: Municipal solid waste. Prognosis. Environmental impacts. Gases emission. Juazeiro do Norte. 


\section{INTRODUÇÃO}

A gestão adequada de Resíduos Sólidos (RS), incluindo os Resíduos Sólidos Urbanos (RSU), é considerada um desafio crucial enfrentado por toda população que busca um desenvolvimento sustentável (GHOSH, 2014). Isso porque, caso nada seja feito nessa direção, o aumento de resíduos não tratados contribuirá significativamente para a intensificação do efeito estufa, da poluição do ar, da poluição das águas e dos problemas de saúde pública (LI et al., 2015; LIU et al., 2018; THAKUR; GANGULY; DHULIA, 2018; YANG et al., 2018).

Em relação ao Brasil, a Associação Brasileira de Empresas de Limpeza Pública e Resíduos Especiais (ABRELPE) divulga anualmente um panorama geral do setor com dados e informações essenciais para entender, planejar e implantar soluções para uma gestão de resíduos mais sustentável (ABRELPE, 2012; 2013; 2014; 2015; 2016; 2017). Nesses relatórios é possível perceber um cenário preocupante em relação a gestão dos RSU de diversas regiões do país. Segundo a ABRELPE (2017), o Brasil gera e coleta, respectivamente, em torno de 214.405 e 195.452 (91\%) toneladas (t) de RSU por dia. Desses resíduos coletados, 81.263 t/dia (41,6\%) são dispostos em lugares impróprios (aterros controlados ou lixões).

Dentre as regiões brasileiras com maiores gerações de resíduos sólidos urbanos, destaca-se o Nordeste. A região é responsável por cerca de 25\% (55.056 t/dia) do total produzido pelo país, caracterizando-se como a segunda maior região geradora de RSU do Brasil. Entretanto, desta quantidade, somente $79 \%$ dos resíduos produzidos são coletados, configurando-se como a menor taxa dentre as cinco regiões brasileiras. Além deste problema, $64,4 \%$ dos RSU coletados são dispostos em lugares impróprios, percentual 22,8\% maior do que a média nacional (ABRELPE, 2017). Nessa região, o estado do Ceará destaca-se como um dos maiores responsáveis pela geração de RSU (17,56\%), estando atrás somente do estado da Bahia $(26,71 \%)$ (ABRELPE, 2016).

Diante da problemática apresentada, verifica-se que a busca por melhores resultados requer a implantação de boas práticas de gestão de RS, que são apontadas em publicações da área acadêmica (FADE, 2014; VAN FAN et al., 2018; GOPU et al., 2018; MAKARICHI; JUTIDAMRONGPHAN; TECHATO, 2018). Contudo, 
para isso, é necessário inicialmente entender mais profundamente a atual situação dos sistemas de gestão e seus resultados. Estudos que caracterizam, diagnosticam e avaliam a atual gestão de resíduos de uma determinada região são frequentes (CHATTOPADHYAY; DUTTA; RAY, 2009; DA SILVA; PIMENTA; CAMPOS, 2013; DICKEL et al., 2018; DINIZ; ABREU, 2018; FEITOSA; DA SILVA, 2018; GHINEA et al., 2016; GOLLAPALLI; KOTA, 2018; KUMAR; SHARMA, 2014; PIAZ; FERREIRA, 2011; YOUNES et al., 2016) e considerados essenciais para o planejamento de futuras melhorias no setor.

Nesse sentido, este estudo teve como objetivo realizar o prognóstico do atual sistema de gestão de resíduos sólidos urbanos do município de Juazeiro do Norte no estado do Ceará. Para alcançar este objetivo, foram utilizados métodos quantitativos de modelagem como intuito de estimar, em um período de 20 anos (2016 a 2035), resultados de ocupação de área por aterro e de emissão de gás metano $\left(\mathrm{CH}_{4}\right)$ e de gás carbônico $\left(\mathrm{CO}_{2}\right)$ causados pelo atual sistema, caso não ocorram mudanças. Espera-se que os resultados obtidos possam auxiliar os tomadores de decisão da região em futuras proposições de intervenções de melhoria e que a abordagem deste estudo possa ser aplicada em outras regiões.

Para melhor organização e compreensão, este estudo foi dividido em seções. Além desta seção de caráter introdutório, a seção 2 (fundamentação teórica) apresenta uma visão geral sobre gestão de RS e a utilização de modelos na previsão de impactos do sistema. A seção 3 é destinada às considerações metodológicas, que abrange as etapas necessárias para a condução da pesquisa. A seção 4 contém os resultados e discussões, apresentando o prognóstico do atual sistema de gestão de resíduos sólidos urbanos do município de Juazeiro do Norte. Por fim, explicitam-se as considerações finais.

\section{FUNDAMENTAÇÃO TEÓRICA}

\subsection{Visão geral da gestão de resíduos sólidos}

Ao longo dos anos, o crescimento da população e a intensificação da economia 
causaram aumento na quantidade de resíduos gerados pelos seres humanos (BRUNNER; RECHBERGER, 2015; GIUSTI, 2009). O aumento dos efeitos negativos trazidos pelos resíduos fez com que surgisse o serviço público de gestão de resíduos em diversas regiões. Primeiramente, as questões higiênicas e de saúde pública eram as prioridades estratégicas e se limitavam às ações de coleta e despejo dos resíduos em lugares mais distantes (BRUNNER; RECHBERGER, 2015; WILSON, 2007). Essa visão evoluiu ao longo dos anos, com o aumento das discussões sobre o assunto, e, atualmente, o objetivo da gestão dos resíduos está bem consolidado na proteção do ser humano e do meio ambiente (BRUNNER; RECHBERGER, 2015).

No Brasil, a fim de mitigar os principais problemas ambientais, sociais e econômicos decorrentes da gestão ineficaz e ineficiente dos resíduos sólidos e criar um ambiente propício para um desenvolvimento sustentável, foi criada a Lei Federal no 12.305/2010, que instituiu a Política Nacional de Resíduos Sólidos (PNRS). A PNRS (BRASIL, 2010) enfatiza que a gestão de RS no país necessita focar principalmente nos seguintes objetivos:

- $\quad$ proteção da saúde pública e da qualidade ambiental;

- incentivo à indústria da reciclagem;

- adoção de tecnologias como forma de minimizar impactos negativos dos RS;

- $\quad$ estímulo à criação de sistemas de gestão de RS otimizados, abrangendo tratamentos de recuperação energética dos resíduos sólidos.

Contudo, para estar alinhado aos objetivos da PNRS e alcançar resultados satisfatórios, o planejamento de um sistema de gestão de resíduos sólidos necessita definir qual é a categoria de resíduo que será focada. Em seguida, deve-se caracterizar e entender a atual situação do sistema através do levantamento de informações, como a gravimetria dos RS, fluxos dos resíduos e os resultados obtidos pelo sistema em ação.

A PNRS (BRASIL, 2010) classifica os resíduos sólidos em 11 categorias e cada uma delas possui características diferentes, demandando planejamentos específicos. Dentre essas categorias, os Resíduos Sólidos Urbanos (RSU) são os resíduos domiciliares somados aos resíduos de limpeza urbana, sendo compostos por 
diferentes tipos de materiais. A gravimetria é uma análise comumente usada em estudos que indicam a quantidade de cada material em um determinado tipo de resíduo. A composição dos resíduos sólidos de uma região é bastante influenciada pela renda da população. Quanto menor a renda, maior é o percentual de material orgânico; por outro lado, quanto maior a renda, menor é a participação de materiais orgânicos e maior é a parcela de materiais recicláveis. A Figura 1 mostra um gráfico da gravimetria dos RS em países com diferentes tipos de renda.

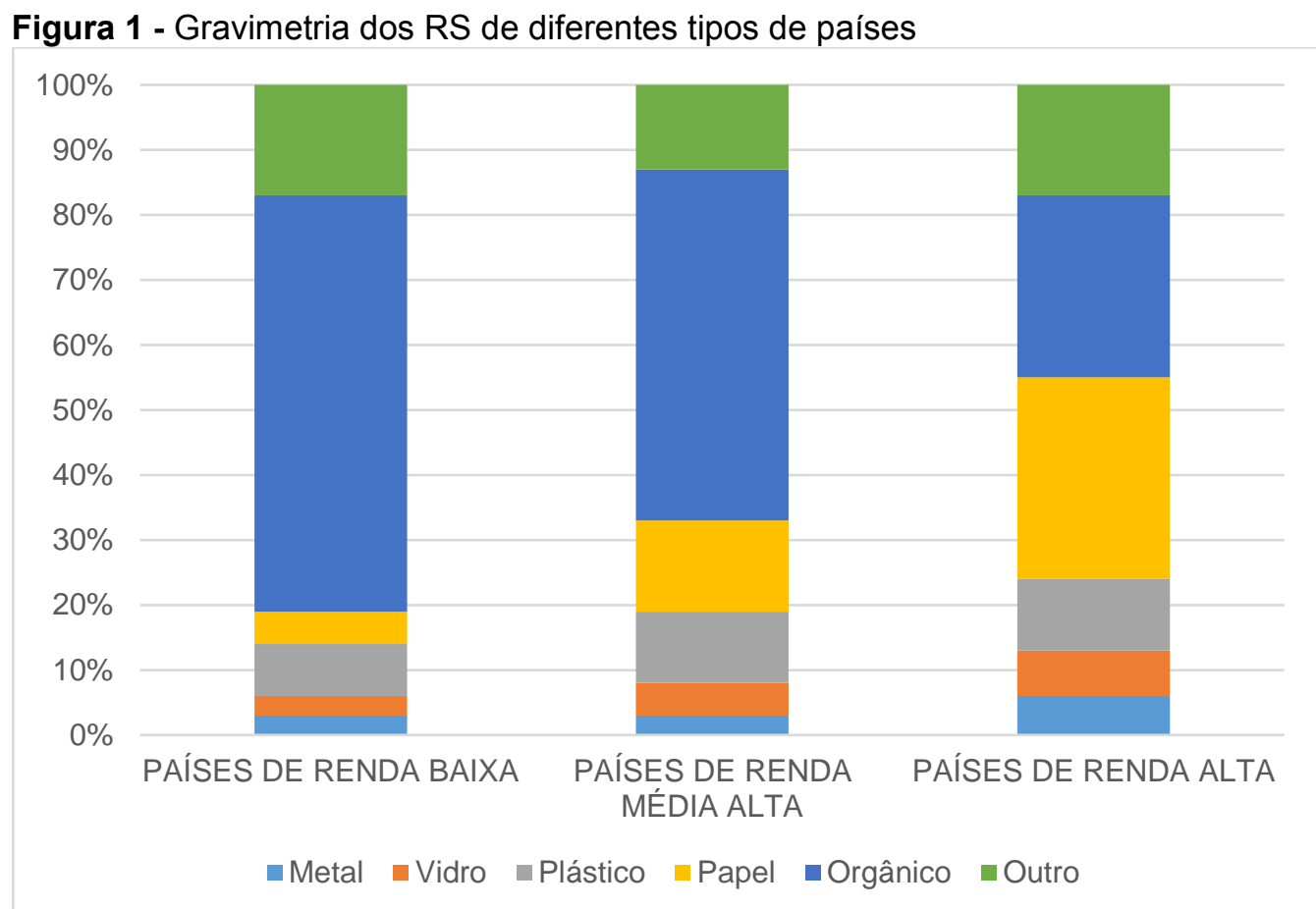

Fonte: World Bank (2012), modificado pelos autores

Segundo a COMLURB (2005) e Chang e Pires (2015), a gravimetria dos RS é essencial para a eficiência do planejamento, do projeto e da operação de um sistema de gestão de resíduos, já que as características físicas e químicas mudam de acordo com o tipo de material. Cabe-se destacar também, que os valores das quantidades de RS gerados e que circulam no sistema são informações básicas necessárias para o planejamento, operação e otimização de sistemas de gestão de resíduos (DEHGHANIFARD; DEHGHANI, 2018; GHINEA et al., 2016).

Quantificar os resíduos presentes em cada etapa do sistema possibilita projetar os resultados da atual gestão e de alternativas de mudanças. Normalmente, os RS seguem um fluxo que se inicia na geração e termina na disposição final. Christensen 
(2011) divide o sistema de gestão de resíduos sólidos nas etapas de geração de resíduos, coleta e transporte, tratamento e, por último, a etapa RUL - reciclagem, utilização e disposição final. Estas etapas podem ser descritas como:

- geração de resíduos - a geração de resíduos é a primeira etapa do sistema e é nela que são definidas características básicas dos resíduos, como categoria, tipo, quantidades e composição;

- coleta e transporte - esta etapa refere-se à separação e armazenamento organizado dos resíduos na origem, aos centros de coletas, à coleta, ao transporte dos RS do ponto de coleta até uma unidade de tratamento ou $\mathrm{RUL}$, e às estações de transferência de resíduos de um veículo menor para um maior;

- tratamento - nesta etapa os RS são tratados através de diferentes tipos de tratamentos (mecânico, térmico ou biológico) com o intuito de recuperar resíduos recicláveis, extrair energia ou alterar certas características dos resíduos (volume, nível de contaminantes etc.) (CHRISTENSEN, 2011);

- RUL (Reciclagem/Utilização/Disposição Final) - tem o objetivo de recuperar e utilizar materiais dos resíduos com algum fim ou depositá-los de forma segura. Na reciclagem, o material virgem é substituído pelo mesmo tipo de material reciclado (como vidro, alumínio, papel e plástico). Já a utilização é o uso de frações dos resíduos ou de resíduos tratados em formas alternativas (como o uso dos RS resultantes do tratamento biológico como fertilizante para terra; uso de frações de plástico e papel como combustível para indústrias de cimento etc.). Por fim, a disposição final deposita os resíduos em aterros.

As quantidades de resíduos de cada uma dessas etapas podem ser definidas através de dados primários, coletados em visita de campo, e/ou dados secundários. Por exemplo, a taxa de geração por unidade (em kg/ano/pessoa) é um parâmetro conveniente e comumente utilizado para projetar a quantidade de RS gerada em uma região (CHRISTENSEN, 2011). 


\subsection{Previsão de impactos da gestão de resíduos sólidos}

Em relação aos impactos dos sistemas de gestão de RS, Chang e Pires (2015) destacam a importância de modelos matemáticos na previsão de resultados futuros, auxiliando na avaliação do atual sistema e na comparação e escolha de alternativas de mudanças. De acordo com Younes et al. (2016), as ferramentas/técnicas de projeção podem ser qualitativas ou quantitativas. As qualitativas utilizam a opinião de especialistas e/ou o julgamento pessoal; as quantitativas, por sua vez, tomam como base os modelos matemáticos. Estes modelos matemáticos podem ser usados, por exemplo, para estimar a receita operacional relativa aos ganhos dos produtos resultantes do sistema de gestão de RS, tais como materiais recicláveis, fertilizantes e energia elétrica (CHANG; PIRES, 2015). Em relação aos resultados ambientais, é possível levantar diferentes pesquisas que utilizam modelos matemáticos para projetar impactos da gestão de RS na emissão de Gases de Efeito Estufa (GEE) e na ocupação de área para disposição final de resíduos ( $\mathrm{CHO}$; $\mathrm{MOON}$; KIM, 2012; CREMIATO et al., 2018; GOLLAPALLI; KOTA, 2018; KARANJEKAR et al., 2015; KUMAR; SHARMA, 2014; YOUNES et al., 2016).

A estimativa das emissões de gases metano $\left(\mathrm{CH}_{4}\right)$ e carbônico $\left(\mathrm{CO}_{2}\right)$ é uma etapa importante no estudo e planejamento do setor. Segundo Turner, Kemp e Williams (2011), as emissões de $\mathrm{CH}_{4}$ e $\mathrm{CO}_{2}$ são responsáveis por $90 \%$ do total de $\mathrm{GEE}$ gerados pelos resíduos. Nesse contexto, a ferramenta LandGEM, criada pela Agência de Proteção Ambiental dos Estados Unidos (USEPA), é comumente usada como modelo de previsão quantitativo da produção de gases a partir dos RSU (CHO; MOON; KIM, 2012; GOLLAPALLI; KOTA, 2018; KARANJEKAR et al., 2015; KUMAR; SHARMA, 2014). Para seu cálculo, é necessário inserir na planilha do LandGEM os valores de i, n, K, Lo, NMOC, volume de metano e Mi. Onde (i) é o ano de início e (n) o ano de término do funcionamento do aterro; (K) é a taxa de geração de metano; (Lo) é a capacidade potencial de geração de metano; (NMOC), em ppmv hexano representa a concentração de compostos orgânicos voláteis não-metânicos; o volume de metano é representado em percentual (\%) e (Mi) é a quantidade de RSU que entra no aterro ou lixão todos os anos. 
A estimativa da ocupação de área para disposição final de resíduos também é essencial no planejamento do sistema de gestão de RS (YOUNES et al., 2016). Os resultados estimados ajudam a dimensionar a abrangência física necessária para 0 funcionamento de aterros, podendo ser comparados com outras soluções que reduzam o volume dos resíduos através de tratamentos, como por exemplo, 0 tratamento térmico. Segundo Younes et al. (2016), o espaço ocupado pelos RSU dispostos em aterro e/ou lixões pode ser estimado através da Equação 01:

$$
\text { Área }=\mathrm{R} \times \mathrm{L} \times \text { Pop } \times 1,5 /(\text { pbulk } \times \mathrm{H})
$$

onde $(R)$ é taxa de geração per capita por ano de RSU (kg/hab/ano), (L) é o tempo de utilização do aterro/lixão (anos), (Pop) é quantidade total de pessoas, (Pbulk) é a densidade dos resíduos em $\mathrm{kg} / \mathrm{m}$, e $(\mathrm{H})$ é a altura das montanhas de resíduos no aterro, dada em metros.

\section{CONSIDERAÇÕES METODOLÓGICAS}

O presente trabalho utilizou métodos quantitativos de modelagem com o intuito de realizar o prognóstico do atual sistema de gestão de RSU de Juazeiro do Norte/CE, caso não haja intervenção de mudanças. Segundo Morabito e Pureza (2012), os modelos quantitativos utilizam linguagem matemática com 0 intuito de estimar resultados de um determinado sistema, podendo ser útil na análise de alternativas e tomada de decisões.

A pesquisa projetou os impactos de área ocupada por aterro e de emissão dos gases metano $\left(\mathrm{CH}_{4}\right)$ e carbônico $\left(\mathrm{CO}_{2}\right)$ em um período de 20 anos (2016 a 2035). A Figura 2 apresenta as quatro etapas seguidas nesta pesquisa. 
Figura 2 - Etapas da pesquisa

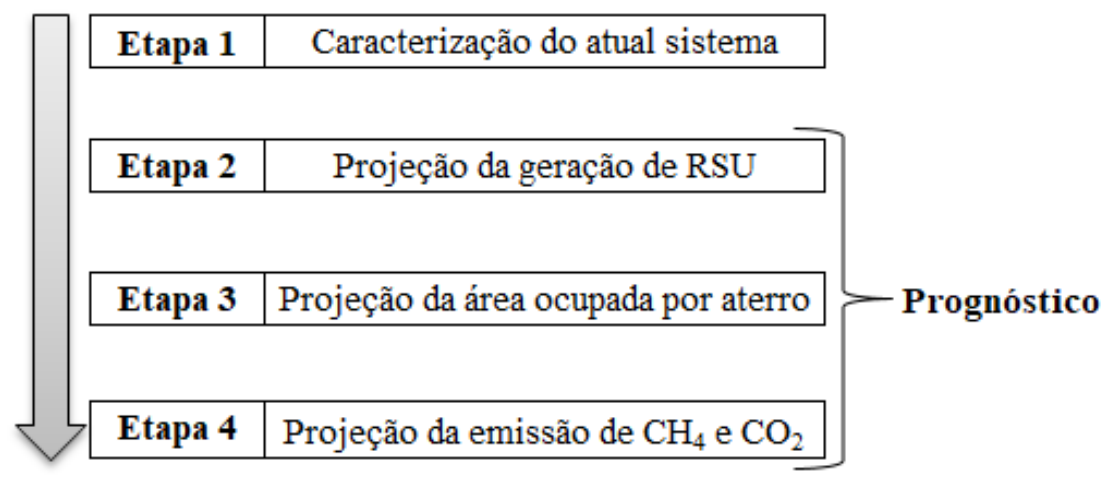

Fonte: Elaboração própria

A primeira etapa teve o objetivo de caracterizar o atual sistema de gestão de RSU de Juazeiro do Norte, levantando e calculando dados relacionados aos fluxos de resíduos que foram úteis nas projeções realizadas nas etapas seguintes. Dados primários foram coletados em uma visita de três dias ao município, realizada em março de 2016. Já os dados secundários, foram levantados nas fontes do IBGE (2015), ABRELPE (2016) e SEMASP (2013). Ainda nessa etapa, esses dados foram utilizados como valores de entrada para calcular as quantidades de RSU gerada, coletada e enterrada no aterro da região no ano de 2015 (ano anterior a visita).

As etapas 2, 3 e 4 estão relacionadas diretamente com o prognóstico desta pesquisa. O prognóstico engloba um período de 20 anos (2016 a 2035) e está dividido nas etapas de "Projeção de geração de RSU", "Projeção da área ocupada por aterro" e "Projeção de emissão de $\mathrm{CH}_{4}$ e $\mathrm{CO}_{2}$ ".

A etapa 2 realizou a projeção das quantidades de RSU gerada, coletada e enterrada no aterro da região em um período de 20 anos. Os dados coletados e calculados na etapa anterior foram utilizados para projetar as quantidades de RSU nos anos de 2016 até 2035. Nessa etapa, foi utilizado o Software Microsoft Exceß $\mathbb{B}^{\text {na }}$ versão 2013, para dar suporte à organização dos dados coletados, à realização de cálculos, e à criação de tabelas, quadros, gráficos e figuras.

A etapa 3 foi responsável pela projeção da área ocupada por aterro. Os resultados desta etapa foram calculados através da Equação (1), apresentada anteriormente, e utilizou parâmetros apontados por Younes et al. (2016). Segundo esses autores, o valor de $800 \mathrm{~kg} / \mathrm{m}^{3}$ é comumente adotado como densidade média dos resíduos ( $\rho_{\text {bulk }}$ ) em diversos estudos na área; o valor da altura das montanhas de 
resíduos $(\mathrm{H})$ deve variar entre 15 e 30 metros (usou-se o valor médio de 22,5 metros); já o tempo médio mais comum de utilização de um aterro/lixão é 20 anos (L).

Por fim, a etapa 4 buscou estimar a quantidade de gás $\mathrm{CH}_{4}$ e CO 2 emitida pelos RSU destinados ao aterro de Juazeiro do Norte ao longo de 20 anos. Esta etapa utilizou a ferramenta LandGEM (USEPA, 2005), cujos parâmetros foram citados anteriormente. Este estudo levou em consideração os critérios da USEPA (2005), que define os valores padrões de $\mathrm{k}=0,05\left(\mathrm{ano}^{-1}\right), \mathrm{L}_{0}=170\left(\mathrm{~m}^{3} \cdot \mathrm{mg}^{-1}\right)$, concentração de $\mathrm{NMOC}=600$ (ppmv hexano), a inexistência de resíduos perigosos e o volume de metano $=50 \%$.

\section{RESULTADOS E DISCUSSÃO}

Esta seção apresenta os resultados referentes as etapas de caracterização do atual sistema de gestão de RSU de Juazeiro do Norte (Etapa 1), Projeção da Geração de RSU (Etapa 2), Projeção da área ocupada por aterro (Etapa 3) e Projeção da emissão de $\mathrm{CH}_{4}$ e $\mathrm{CO}_{2}$ (Etapa 4).

\subsection{Etapa 1 - Caracterização do atual sistema}

O município de Juazeiro do Norte está localizado no sul do estado do Ceará e, segundo uma estimativa do IBGE (2015), possuía em torno de 266.022 habitantes no final de 2015. Na visita ao município em 2016, foi constatado que não há um estudo específico que estime a quantidade de resíduos sólidos urbanos gerados na região. Por esse motivo, o presente estudo utilizou a taxa de geração por unidade (kg.hab1.ano-1) sugerido por Christensen (2011) para estimar a sua geração de RSU. A Tabela 1 detalha os dados base para o cálculo e a quantidade de RSU gerada no ano de 2015. 
Tabela 1 - Cálculo da geração de RSU em Juazeiro do Norte em 2015

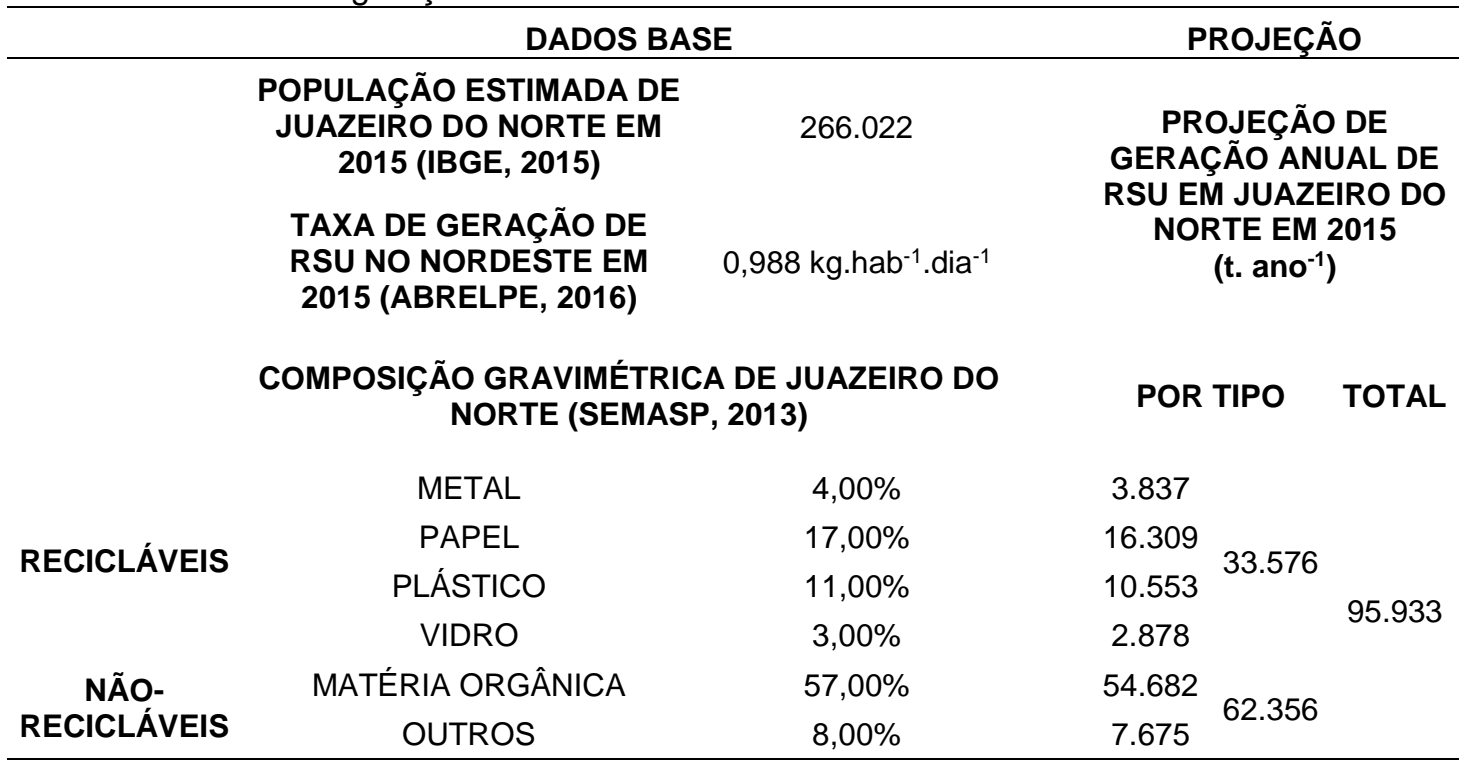

Fonte: Elaboração com base nos dados coletados e calculados na pesquisa.

No cálculo, considerou-se que a taxa de geração da região é igual à taxa média do Nordeste de 0,988 kg.hab-1.ano-1 (ABRELPE, 2016). Logo, multiplicando essa taxa pelos 266.022 habitantes de Juazeiro do Norte (IBGE, 2015), estima-se que o município gerou aproximadamente um total de 263 toneladas (t) de RSU por dia, ou seja, 95.933 t no ano de 2015. Destas 95.933 toneladas, considerando a composição gravimétrica do município (SEMASP, 2013), 3.837 t são metal, 16.309 t papel, 10.553 t plástico, 2.878 t vidro, 54.682 t matéria orgânica e 7.675 t são outros tipos de materiais.

Em relação a coleta e disposição dos RSU gerados, os funcionários do aterro da região (aterro da Palmeirinha) estimam que são coletadas, transportadas e despejadas, aproximadamente, 235 t de RSU por dia. Até o momento da visita, o sistema de gestão de RSU do município de Juazeiro do Norte não possuía unidades de tratamento de resíduos. Como o único destino dos resíduos coletados é o aterro, calcula-se que em torno de 89\% (235/263) dos RSU gerados em Juazeiro do Norte são coletados e dispostos no aterro da região. Segundo os funcionários do aterro, cerca de $20 \%$ do que é depositado (47 t/dia) passam por triagem e são vendidos pelos catadores e o restante (188 t/dia) é enterrado no próprio aterro.

Em resumo, do total de RSU gerado no município, aproximadamente $89 \%$ são coletados e depositados no aterro controlado de Palmeirinha. Desses 89\%, 18\% 
passam por triagem e são vendidos para reciclagem. O restante, $71 \%$ do RSU gerado, são enterrados no aterro do município (Tabela 2).

Tabela 2 - Fluxos de RSU em Juazeiro do Norte em 2015

\begin{tabular}{ccc}
\hline RSU Gerado & RSU Coletado & RSU Coletado e Enterrado no aterro \\
\hline $263 \mathrm{t} / \mathrm{dia}$ & $235 \mathrm{t} / \mathrm{dia}$ & $188 \mathrm{t} / \mathrm{dia}$ \\
$100 \%$ & $89 \%$ & $71 \%$ \\
\hline Fonte: Elaboracão com base nos dados coletados e calculados na pesquisa
\end{tabular}

Fonte: Elaboração com base nos dados coletados e calculados na pesquisa

\subsection{Etapa 2 - Projeção da geração de RSU}

A Figura 3 mostra a projeção da quantidade de RSU gerada em Juazeiro do Norte em um período de 20 anos (2016 até 2035). Esses valores são dados de entrada essenciais para projetar os resultados do sistema em estudo. $O$ cálculo desses valores considerou uma taxa média de crescimento anual de geração de RSU no Ceará $(2,4 \%)$ e partiu, com valor base, da quantidade de RSU gerada em 2015 que foi calculado e apresentado anteriormente na Tabela 2. O valor médio de $2,4 \%$ foi calculado através das taxas dos anos de 2010 até 2015 (última taxa disponível até o momento da pesquisa), apontadas pela ABRELPE (2012; 2013; 2014; 2015; 2016).

Estima-se que a geração de RSU de Juazeiro do Norte entre 2015 até 2035 cresça de 95.933 para 154.158 toneladas por ano, ou seja, um aumento de $60,7 \%$ nos próximos 20 anos. Como a composição gravimétrica foi mantida constante nesta projeção, a matéria orgânica continuará sendo o principal tipo de RSU produzido pela região, chegando a um total de 87.870 toneladas no ano de 2035; no mesmo ano, serão geradas 53.955 e 12.333 toneladas de resíduos recicláveis e de outros resíduos, respectivamente. 
Figura 3 - Projeção de geração de RSU em Juazeiro do Norte de 2016 a 2035 (t.ano-1)

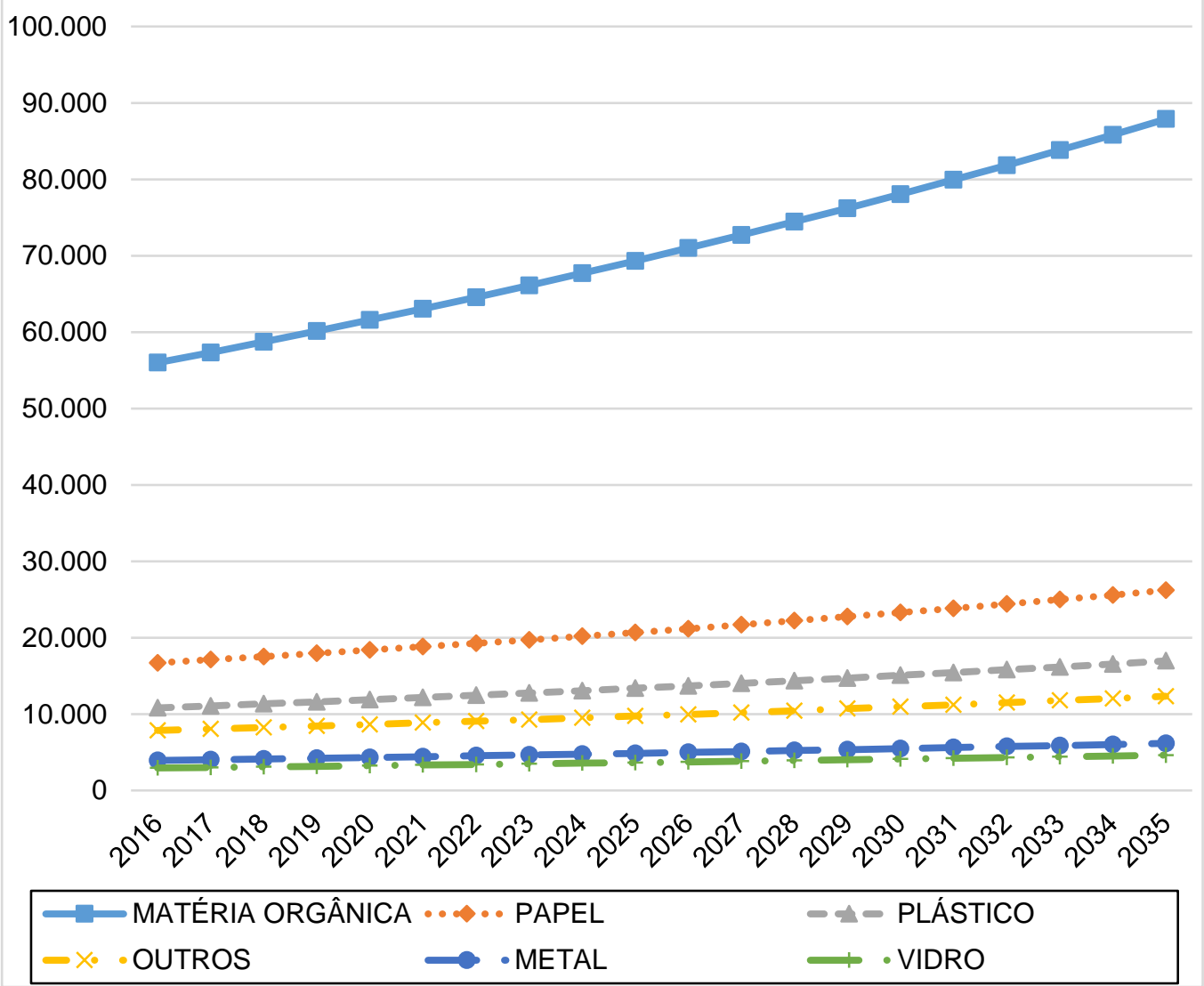

Fonte: Elaboração com base nos dados coletados e calculados na pesquisa

\subsection{Etapa 3 - Projeção da área ocupada por aterro}

Para o cálculo da área ocupada pelos resíduos sólidos urbanos gerados nos anos de 2016 a 2035, utilizou-se a Equação 1 apresentada por Younes et al. (2016). Na pesquisa, contabilizou-se somente o RSU coletado e enterrado (Tabela 3) que foi calculado levando em consideração o percentual apontado na etapa 1 (71\%).

Em relação a taxa de geração per capita de $\mathrm{RSU}(\mathrm{R})$, utilizou-se a média anual de habitantes no período de projeção (média anual de 280.266 habitantes), calculada a partir das taxas de crescimento populacional do Ceará do IBGE (2015); e a geração anual média (124.214 toneladas por ano), calculada através dos dados projetados anteriormente na etapa 2. A taxa de geração per capita encontrada foi de 443 kg.hab${ }^{1}$.ano $^{-1}$, mas como somente $71 \%$ é coletado e enterrado, foi utilizada na projeção a taxa de $315 \mathrm{~kg} \cdot \mathrm{hab}^{-1} \cdot \mathrm{ano}^{-1}$. 
Tabela 3 - Projeção de RSU gerado, e coletado e enterrado no aterro de Juazeiro do Norte (t.ano ${ }^{-1}$ )

\begin{tabular}{|c|c|c|c|c|c|c|c|}
\hline \multicolumn{2}{|r|}{2016} & \multicolumn{2}{|c|}{2017} & \multicolumn{2}{|r|}{2018} & \multicolumn{2}{|c|}{2019} \\
\hline Gerado & $\begin{array}{l}\text { Coletado e } \\
\text { Enterrado }\end{array}$ & Gerado & $\begin{array}{l}\text { Coletado e } \\
\text { Enterrado }\end{array}$ & Gerado & $\begin{array}{l}\text { Coletado e } \\
\text { Enterrado }\end{array}$ & Gerado & $\begin{array}{l}\text { Coletado e } \\
\text { Enterrado }\end{array}$ \\
\hline 98.235 & 69.747 & 100.593 & 71.421 & 103.007 & 73.135 & 105.479 & 74.890 \\
\hline \multicolumn{2}{|r|}{2020} & \multicolumn{2}{|c|}{2021} & \multicolumn{2}{|r|}{2022} & \multicolumn{2}{|c|}{2023} \\
\hline Gerado & $\begin{array}{l}\text { Coletado e } \\
\text { Enterrado }\end{array}$ & Gerado & $\begin{array}{l}\text { Coletado e } \\
\text { Enterrado }\end{array}$ & Gerado & $\begin{array}{l}\text { Coletado e } \\
\text { Enterrado }\end{array}$ & Gerado & $\begin{array}{l}\text { Coletado e } \\
\text { Enterrado }\end{array}$ \\
\hline 108.011 & 76.688 & 110.603 & 78.528 & 113.258 & 80.413 & 115.976 & 82.343 \\
\hline \multicolumn{2}{|r|}{2024} & \multicolumn{2}{|c|}{2025} & \multicolumn{2}{|r|}{2026} & \multicolumn{2}{|c|}{2027} \\
\hline Gerado & $\begin{array}{l}\text { Coletado e } \\
\text { Enterrado }\end{array}$ & Gerado & $\begin{array}{l}\text { Coletado e } \\
\text { Enterrado }\end{array}$ & Gerado & $\begin{array}{l}\text { Coletado e } \\
\text { Enterrado }\end{array}$ & Gerado & $\begin{array}{l}\text { Coletado e } \\
\text { Enterrado }\end{array}$ \\
\hline 118.759 & 84.319 & 121.609 & 86.343 & 124.528 & 88.415 & 127.517 & 90.537 \\
\hline \multicolumn{2}{|r|}{2028} & \multicolumn{2}{|c|}{2029} & \multicolumn{2}{|r|}{2030} & \multicolumn{2}{|c|}{2031} \\
\hline Gerado & $\begin{array}{l}\text { Coletado e } \\
\text { Enterrado }\end{array}$ & Gerado & $\begin{array}{l}\text { Coletado e } \\
\text { Enterrado }\end{array}$ & Gerado & $\begin{array}{l}\text { Coletado e } \\
\text { Enterrado }\end{array}$ & Gerado & $\begin{array}{l}\text { Coletado e } \\
\text { Enterrado }\end{array}$ \\
\hline 130.577 & 92.710 & 133.711 & 94.935 & 136.920 & 97.213 & 140.206 & 99.546 \\
\hline \multicolumn{2}{|r|}{2032} & \multicolumn{2}{|c|}{2033} & \multicolumn{2}{|r|}{2034} & \multicolumn{2}{|c|}{2035} \\
\hline Gerado & $\begin{array}{l}\text { Coletado e } \\
\text { Enterrado }\end{array}$ & Gerado & $\begin{array}{l}\text { Coletado e } \\
\text { Enterrado }\end{array}$ & Gerado & $\begin{array}{l}\text { Coletado e } \\
\text { Enterrado }\end{array}$ & Gerado & $\begin{array}{l}\text { Coletado e } \\
\text { Enterrado }\end{array}$ \\
\hline 143.571 & 101.935 & 147.017 & 104.382 & 150.545 & 106.887 & 154.158 & 109.452 \\
\hline
\end{tabular}

Fonte: Elaboração com base nos dados coletados e calculados na pesquisa.

Os valores dos parâmetros L (20 anos), $\rho_{\text {bulk }}\left(800 \mathrm{~kg} / \mathrm{m}^{3}\right)$ e $\mathrm{H}$ (valor médio de 22,5 metros) foram definidos através das recomendações do estudo de Younes et al. (2016). Logo, o cálculo da área ocupada para a disposição de resíduos sólidos urbanos no aterro controlado em Juazeiro do Norte é de:

$$
\begin{gathered}
\text { Área }=315 \times 20 \times 280.266 \times 1,5 /(800 \times 22,5) ; \\
\text { Área }=147.140 \mathrm{~m}^{2}=14,71 \text { hectares } .
\end{gathered}
$$

Ao final do cálculo, pode-se inferir que, caso o atual nível de desempenho de gestão se mantenha, os RSU coletados e enterrados em Juazeiro do Norte, nos próximos 20 anos, ocuparão uma área total equivalente ao espaço ocupado por, aproximadamente, 20 campos de futebol.

\subsection{Etapa 4 - Projeção da emissão de $\mathrm{CH}_{4}$ e $\mathrm{CO}_{2}$}

Conforme já mencionado, utilizou-se o modelo da USEPA (2005) - chamado de LandGEM - para projetar as emissões de gás metano e gás carbônico gerados pelos resíduos enterrados no aterro. Esta pesquisa adotou os critérios da USEPA (2005), 
que define os valores padrões de $\mathrm{k}\left(0,05 \mathrm{ano}^{-1}\right)$, Lo $\left(170 \mathrm{~m}^{3} \cdot \mathrm{mg}^{-1}\right)$, concentração de NMOC (600 ppmv hexano) e volume de metano (50\%). Em relação a quantidade de RSU enterrada no aterro $\left(\mathrm{M}_{\mathrm{i}}\right)$ foi inserido os valores encontrados com base na Tabela 3 apresentado na etapa 3. Ao final da aplicação do modelo LandGEM, chegou-se aos valores de emissão de gases mostrados na Figura 4.

Figura 4 - Estimativa de geração de $\mathrm{CH}_{4}$ e $\mathrm{CO}_{2}$ a partir dos $\mathrm{RSU}$ enterrados nos próximos 20 anos em Juazeiro do Norte (t.ano ${ }^{-1}$ )

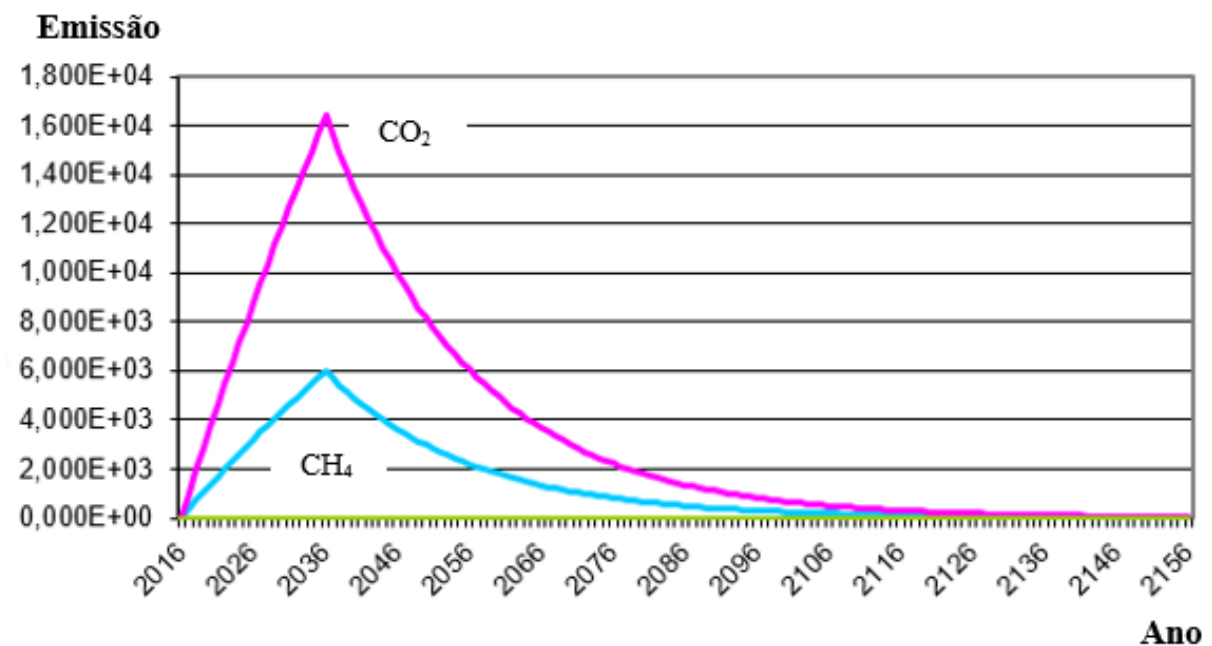

Fonte: Saída da ferramenta LandGEM

Como no aterro de Juazeiro do Norte não existe a captura dos gases gerados a partir dos RSU, considerou-se que todo o gás produzido é disposto no ambiente. Logo, estima-se que, nos próximos 20 anos, o RSU enterrado no aterro emitirá um total aproximado de 72.110 toneladas de gás metano (média de 3.605 toneladas por ano) e 197.800 toneladas de gás carbônico (média de 9.890 toneladas por ano). Além disso, constata-se que, mesmo depois de desativado no final do ano de 2035, o aterro ainda será um gerador de GEE por várias décadas, chegando a emitir um total de 153.300 toneladas de gás metano e 420.600 toneladas de gás carbônico entre os anos de 2016 a 2056, provenientes dos RSU coletados e enterrados entre 2016 e 2035.

Calcula-se que, ao dividir o total da emissão pela quantidade de anos que foram gerados esses resíduos (20 anos), os RSU coletados e enterrados entre 2016 e 2035 sejam responsáveis anualmente pela emissão de, em média, 7.665 toneladas de gás metano e 21.030 toneladas de gás carbônico. 


\section{CONSIDERAÇÕES FINAIS}

Para esta pesquisa, os dados e informações coletadas se mostraram suficientes para alcançar o objetivo proposto. Obteve-se uma visão geral do atual sistema de gestão de Resíduos Sólidos Urbanos (RSU) de Juazeiro do Norte por meio da quantificação dos fluxos de RSU gerados, coletados e enterrados no aterro da região. Esses valores de RSU foram essenciais para a projeção de impactos que compuseram o prognóstico apresentado.

As projeções dos resultados do prognóstico utilizaram a taxa média de crescimento anual de geração de RSU no Ceará $(2,4 \%)$ e o percentual de RSU enterrado no aterro do município (71\%). Esses valores foram inseridos em modelos matemáticos comumente utilizados em pesquisas de RS e, ao final, foram projetados valores relacionados à área ocupada por aterro e à emissão dos gases $\mathrm{CH}_{4} \mathrm{e} \mathrm{CO}_{2}$. Os resultados do prognóstico apontam aumento de 60,7\% na geração anual de RSU em um período de 20 anos (2016 até 2035) e, caso nada seja feito, os resíduos coletados e enterrados no aterro nesse período ocuparão uma área equivalente a 20 campos de futebol (147.140 $\mathrm{m}^{2}$ ) e emitirão aproximadamente 153.300 toneladas de gás metano e 420.600 toneladas de gás carbônico que se estenderão por 40 anos.

Os resultados desta pesquisa destacam um cenário futuro preocupante que pode e deve ser mitigado através do estudo, planejamento e implementação de alternativas de mudanças do atual sistema de gestão de resíduos da região. Nesse sentido, o aumento da reciclagem e a utilização de tecnologias de tratamentos de resíduos, além de estarem de acordo com a (PNRS), otimizariam os resultados de ocupação de área e de emissão dos gases $\mathrm{CH}_{4}$ e $\mathrm{CO}_{2}$. Por exemplo, dados da FEAM (2010) apontam que cerca de $72 \%$ dos RSU do Japão são tratados através de tecnologias térmicas, diminuindo o volume dos resíduos em até 90\% (LOMBARDI; CARNEVALE; CORTI, 2015). Além disso, estima-se que somente na Europa as usinas de tratamento de RS processem cerca de 81 milhões de toneladas de RS por ano, evitando, em média, a emissão de 30 milhões de toneladas de $\mathrm{CO}_{2}$ (CEWEP, 2013).

Destaca-se que o estudo utilizou a taxa de geração de RSU per capita do estado do Ceará, afetando a precisão da projeção dos cenários. Não foram 
encontrados estudos que apontem parâmetros precisos de produção de RSU em Juazeiro do Norte. Sugere-se que trabalhos futuros realizem um levantamento primário com uma amostra significativa para definir valores e características mais realistas e atuais da geração de RSU da região. Ademais, pesquisas futuras podem ser realizadas com o intuito de comparar os resultados obtidos no prognóstico deste estudo com projeções de impactos vinculados a proposições de melhoria para a problemática em estudo (ex.: utilização de unidades de tratamento de resíduos).

\section{REFERÊNCIAS}

ABRELPE - Associação Brasileira de Empresas de Limpeza Pública e Resíduos Especiais. Panorama dos resíduos sólidos no Brasil 2011. 2012. São Paulo, SP.

ABRELPE - Associação Brasileira de Empresas de Limpeza Pública e Resíduos Especiais. Panorama dos resíduos sólidos no Brasil 2012. 2013. São Paulo, SP.

ABRELPE - Associação Brasileira de Empresas de Limpeza Pública e Resíduos Especiais. Panorama dos resíduos sólidos no Brasil 2013. 2014. São Paulo, SP.

ABRELPE. Associação Brasileira de Empresas de Limpeza Pública e Resíduos Especiais. Panorama dos resíduos sólidos no Brasil 2014. 2015. São Paulo, SP.

ABRELPE. Associação Brasileira de Empresas de Limpeza Pública e Resíduos Especiais. Panorama dos resíduos sólidos no Brasil 2015. 2016. São Paulo, SP.

ABRELPE. Associação Brasileira de Empresas de Limpeza Pública e Resíduos Especiais. Panorama dos resíduos sólidos no Brasil 2016. 2017. São Paulo, SP.

BRASIL. Lei no 12.305, de 2 de agosto de 2010. Institui a Política Nacional de Resíduos Sólidos; altera a Lei no 9.605, de 12 de fevereiro de 1998; e dá outras providências. Diário Oficial da União, 3 de agosto de 2010. Seção 1, Atos do Poder Legislativo. Imprensa Nacional.

BRUNNER, P. H.; RECHBERGER, H. Waste to energy - key element for sustainable waste management. Waste Management, v. 37, p. 3 - 12, 2015.

https://doi.org/10.1016/i.wasman.2014.02.003

CEWEP. Confederation of European Waste to Energy Plants. Heating and lighting the way to sustainable future: energy efficiency and climate protection. 2013. Disponível em: http://www.cewep.eu/m_1073. Acesso em: 16 maio 2017.

CHANG, N. B.; PIRES, A. Sustainable solid waste management: a systems engineering approach. New Jersey: IEEE Wiley, 2015. https://doi.org/10.1002/9781119035848

CHATTOPADHYAY, S.; DUTTA, A.; RAY, S. Municipal solid waste management in Kolkata, India - a review. Waste Management, v. 29, n. 4, p. 1449 -1458, 2009.

https://doi.org/10.1016/j.wasman.2008.08.030 
$\mathrm{CHO}, \mathrm{H}$. S.; MOON, H. S.; KIM, J. Y. Effect of quantity and composition of waste on the prediction of annual methane potential from landfills. Bioresource Technology, v. 109, p. 86 - 92, 2012. https://doi.org/10.1016/i.biortech.2012.01.026

CHRISTENSEN, T. H. Solid waste technology and management. Chichester, UK: John Wiley \& Sons. 2011. https://doi.org/10.1002/9780470666883

COMLURB. Companhia Municipal de Limpeza Urbana. Caracterização gravimétrica dos resíduos sólidos domiciliares do município do Rio de Janeiro. Prefeitura do Rio de Janiero. 2005. Disponível em: http://www.rio.rj.gov.br/comlurb. Acesso em: 11 maio 2016.

CREMIATO, R. et al. Environmental impact of municipal solid waste management using Life Cycle Assessment: The effect of anaerobic digestion, materials recovery and secondary fuels production. Renewable Energy, v. 124, p. 180 -188, 2018.

https://doi.org/10.1016/j.renene.2017.06.033

DA SILVA, L. A. A.; PIMENTA, H. C. D.; CAMPOS, L. M. DE S. C. Logística reversa dos resíduos eletrônicos do setor de informática : realidade, perspectivas e desafios na cidade do Natal-RN. Revista Produção Online, v. 13, n. 2, p. 544-576, 2013.

https://doi.org/10.14488/1676-1901.v13i2.1133

DEHGHANIFARD, E.; DEHGHANI, M. H. Evaluation and analysis of municipal solid wastes in Tehran, Iran. MethodsX, v. 5, n. February, p. 312-321, 2018.

https://doi.org/10.1016/j.mex.2018.04.003

DICKEL, P. R. G. et al. Uso da logística reversa para descarte de lâmpadas fluorescentes à luz da pnrs: o caso do instituto federal de educação do Rio Grande do Sul. Revista Produção Online, v. 18, n. 1, p. 265-284, 2018. https://doi.org/10.14488/16761901.v18i1.2766

DINIZ, G. M.; ABREU, M. C. S. DE. Disposição (ir)responsável de resíduos sólidos urbanos no estado do Ceará: desafios para alcançar a conformidade legal. Revista de gestão social e ambiental, v. 12, n. 2, p. 21-38, 2018. https://doi.org/10.24857/rgsa.v12i2.1412

FADE. Análise das diversas tecnologias de tratamento e disposição final de resíduos sólidos urbanos no Brasil , Europa , Estados Unidos e Japão. Grupo de resíduos sólidos, UFPE/BNDES. 2014.

FEAM. Fundação Estadual de Meio Ambiente. Estudo do estado da arte e análise de viabilidade técnica, econômica e ambiental da implantação de uma usina de tratamento térmico de resíduos sólidos urbanos com geração de energia elétrica no estado de Minas Gerais: relatório 1 - estado da arte do tratamento térmico de resíduos sólidos urbanos com geração de energia elétrica. 2.ed. Belo Horizonte. 2010.

FEITOSA, I. S. C. DE S.; DA SILVA, G. L. Análise do processo produtivo e capacidade operacional de uma organização de coleta seletiva de resíduos sólidos. Revista Produção Online, v. 18, n. 4, p. 1344-1373, 2018. https://doi.org/10.14488/1676-1901.v18i4.2945

GHINEA, C. et al. Forecasting municipal solid waste generation using prognostic tools and regression analysis. Journal of Environmental Management, v. 182, p. 80-93, 2016. https://doi.org/10.1016/j.jenvman.2016.07.026 
GHOSH, S. K. Paradigm shift for a total waste management in developing in India. In: Proceedings of the fourth international conference on solid waste management. p. 312. 2014.

GIUSTI, L. A review of waste management practices and their impact on human health. Waste Management, v. 29, n. 8, p. 2227-2239, 2009.

https://doi.org/10.1016/j.wasman.2009.03.028

GOLLAPALLI, M.; KOTA, S. H. Methane emissions from a landfill in north-east India:

Performance of various landfill gas emission models. Environmental Pollution, v. 234, p. 174-180, 2018. https://doi.org/10.1016/j.envpol.2017.11.064

GOPU, C. et al. Valorizing municipal solid waste: Waste to energy and activated carbons for water treatment via pyrolysis. Journal of Analytical and Applied Pyrolysis, v. 133, p. 4858, 2018. https://doi.org/10.1016/j.jaap.2018.05.002

IBGE. Instituto Brasileiro de Geografia e Estatística. Estimativas populacionais para os municípios e para as unidades da federação brasileiras em 01.07.2015. 2015.

Disponível em: ftp://ftp.ibge.gov.br/Estimativas_de_Populacao /Estimativas_2015/estimativa _dou_2015_20150915.xls. Acesso em: 05 out. 2015.

KARANJEKAR, R. V. et al. Estimating methane emissions from landfills based on rainfall, ambient temperature, and waste composition: the CLEEN model. Waste Management, v. 46, p. 389-398, 2015. https://doi.org/10.1016/j.wasman.2015.07.030

KUMAR, A.; SHARMA, M. P. Estimation of GHG emission and energy recovery potential from MSW landfill sites. Sustainable Energy Technologies and Assessments, v. 5, p. 5061, 2014. https://doi.org/10.1016/j.seta.2013.11.004

$\mathrm{LI}$, Y. et al. Waste incineration industry and development policies in China. Waste Management, v. 46, p. 234-241, 2015. https://doi.org/10.1016/j.wasman.2015.08.008

LIU, Y. et al. Odor impact assessment of trace sulfur compounds from working faces of landfills in Beijing, China. Journal of Environmental Management, v. 220, n. January, p. 136-141, 2018. https://doi.org/10.1016/j.jenvman.2018.04.122

LOMBARDI, L.; CARNEVALE, E.; CORTI, A. A review of technologies and performances of thermal treatment systems for energy recovery from waste. Waste Management, v. 37, p. 26-44, 2015. https://doi.org/10.1016/j.wasman.2014.11.010

MAKARICHI, L.; JUTIDAMRONGPHAN, W.; TECHATO, K. ANAN. The evolution of wasteto-energy incineration: A review. Renewable and Sustainable Energy Reviews, v. 91, p. 812-821, 2018. https://doi.org/10.1016/j.rser.2018.04.088

MORABITO NETO, R.; PUREZA, V. Modelagem e Simulação. In: MIGUEL, P. A. C. (Org.). Metodologia de Pesquisa em Engenharia de Produção e Gestão de Operações. Rio de Janeiro: Elsevier, p. 169 - 198, 2012.

PIAZ, J. F. D.; FERREIRA, G. M. V. Gestão de resíduos sólidos domiciliares urbanos: o caso do município de Marau - RS. Revista de gestão social e ambiental, v. 5, n. 1, p. 33-47, 2011. https://doi.org/10.5773/rgsa.v5i1.248

SEMASP. Secretaria de Meio Ambiente Agricultura e Serviços Públicos. Plano de gestão 
integrada de resíduos sólidos urbanos - PGIRSU. Secretaria de Meio Ambiente Agricultura e Serviços Públicos Secretaria de Recursos Hídricos e Ambiente Urbano. Juazeiro do Norte, Ceará. 2013.

THAKUR, P.; GANGULY, R.; DHULIA, A. Occupational Health Hazard Exposure among municipal solid waste workers in Himachal Pradesh, India. Waste Management, v. 78, p. 483-489, 2018. https://doi.org/10.1016/j.wasman.2018.06.020

TURNER, D. A.; KEMP, S.; WILLIAMS, I. Carbon footprinting in the UK waste management sector. Carbon Management, v. 2, n. 6, p. 677-690, 2011. https://doi.org/10.4155/cmt.11.67

USEPA. United States Environmental Protection Agency. Landfill gas emissions model v.3.02. 2005. Disponível em: http://www.epa.gov/ttn/catc/dir1/landgem-v302.xls. Acesso em: 09 dez. 2015.

VAN FAN, Y. et al. Anaerobic digestion of municipal solid waste: energy and carbon emission footprint. Journal of environmental management, v. 223, p. 888-897, 2018. https://doi.org/10.1016/j.jenvman.2018.07.005

WILSON, D. C. Development drivers for waste management. Waste Management and Research, v. 25, n. 3, p. 198-207, 2007. https://doi.org/10.1177/0734242X07079149

WORLD BANK. Countries: página institucional. 2014. Disponível em: http://www.worldbank.org/en/country. Acesso em: 02 maio 2017.

YANG, D. et al. Inventories and reduction scenarios of urban waste-related greenhouse gas emissions for management potential. Science of the Total Environment, v. 626, p. 727736, 2018. https://doi.org/10.1016/j.scitotenv.2018.01.110

YOUNES, M. K. et al. Landfill area estimation based on integrated waste disposal options and solid waste forecasting using modified ANFIS model. Waste Management, v. 55, p. 311, 2016. https://doi.org/10.1016/i.wasman.2015.10.020

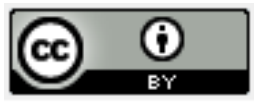

Artigo recebido em: 20/01/2019 e aceito para publicação em: 01/12/2019 DOI: http://dx.doi.org/10.14488/1676-1901.v19i4.3550 